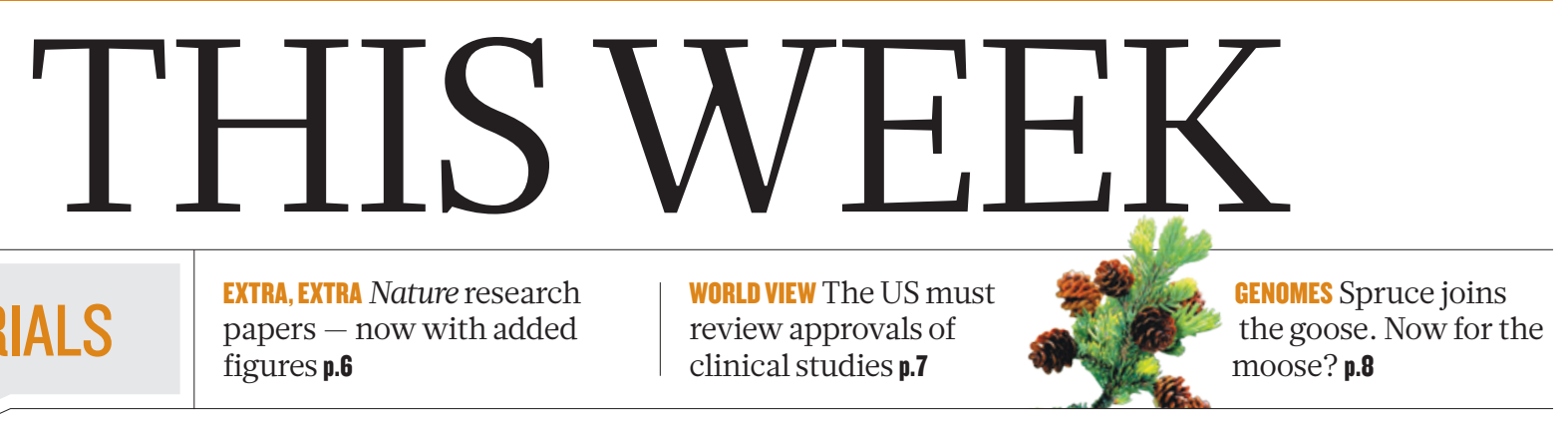

EDITORIALS figures $\mathbf{p . 6}$
WORLD VIEW The US must clinical studies $\mathbf{p . 7}$ the goose. Now for the moose? p.8

\title{
The paper trail
}

\section{Scientists must embrace funding-agency efforts to track research outputs and encourage} open access to the literature.

$\mathrm{L}$ ast week, the world's research-funding agencies signalled a welcome desire for wider access to published papers. The Global Research Council, a voluntary discussion forum that takes input from hundreds of funding agencies in regional meetings around the globe, released an action plan for promoting open access - although specific policies are left up to individual agencies (go.nature.com/ gonk6w).

Scientists need this top-down push. Individually, they have proved reluctant to make their papers freely available, despite the determined efforts of open-access campaigners. For example, although the Wellcome Trust in London, one of the world's biggest biomedical research charities, has since 2005 provided an open-access mandate and the money to support it, by last June only $55 \%$ of the research papers that it funded were open access. Most of those had been uploaded into repositories by publishers, rather than by researchers.

The Global Research Council discussions and action plan have made it clear that when push comes to shove, most agencies lack the will to fund 'gold' open access - in which the author pays for a paper to be free to access as soon as it is published. The UK funding councils, which all provide money towards gold open access, are notable exceptions. Others, such as agencies in the United States, have little money to spare, and are agreeing to wait for publishers or authors to make the results of research freely available six months or a year after publication - 'green' open access. Germany has a dual system, in which researchers can apply for funds to make papers immediately available, but universities can also apply for pots of money expressly to support open access. In Brazil, a system whereby the government negotiates with publishers for open access on a national level is being considered. The permutations are endless.

There is no consensus on whether funding agencies should merely encourage researchers to make their work open (through either green or gold routes), or should actively monitor progress and provide sanctions - such as refusing future grants - for non-compliance. To some extent, reluctance to enforce mandates comes from a desire to ease scientists into open access slowly, but it can also signal endorsement of the idea that agencies should just give researchers opportunities and support for open access, ultimately leaving scientists the freedom to do what they want with their papers.

There is one thing on which funding agencies agree, however. To monitor whether open-access mandates are effective, and to share information on those that are, agencies need to track the outputs of their funding better. At the moment, only a few funders, mainly medical ones - such as the UK Medical Research Council, the Wellcome Trust and the US National Institutes of Health - can give a figure for the proportion of papers resulting from their funding that are open access.

So publishers and funding agencies alike are jumping at new ways to track the sources of funding for published scholarly research. In the same week as the Global Research Council released its action plan,

the non-profit publisher alliance CrossRef launched a service called FundRef (www.crossref.org/fundref). The initiative provides a standardized way to report funding sources for published research, by adding them to the metadata on online research papers.

In the United Kingdom, funding agencies expect researchers to provide extensive details on the results of their funding through

"To monitor

whether open-

access mandates

are effective,

agencies need

to track the

outputs of their

funding." mechositories through mechanisms such as re3data.org, a registry of repositories. At the same time, the ORCID system provides unique identifying numbers to track individual researchers' work, and services such as Figshare are helping to make other types of output, such as data, recognizable and citable.

All this means that as funding agencies push for open access, researchers will need to have their outputs tracked as never before. They should embrace this as a chance both to show off their publications and to acknowledge the support networks that fund their work.

\section{Moral authority}

Research must be seen to be accountable, even if that means hanging on to redundant reviews.

A ll scientists must contend with regulation and bureaucracy, despite their frequent complaints that such processes stifle and slow their work. US researchers in gene therapy perhaps feel the pressure of red tape more than most. Is now the right time to ease that burden?

The US Institute of Medicine wants to find out. This week, it kicked off a review of an oversight committee that many in gene therapy argue is redundant. They might be right, but when it comes to medical ethics, it is not enough for scientists to do the right thing - they must also be seen to do so.

The Recombinant DNA Advisory Committee (RAC) was set up within the National Institutes of Health (NIH) in 1974 as a direct response to public concerns about the ethics and safety of research involving lab-assembled DNA sequences. After devising guidelines 
for this research, the committee gained the power to approve or reject proposed experiments in humans. As the field has grown, so has the number of experiments that must be given the green light by the RAC, including those that fall under the umbrella of gene therapy.

The problem, as gene therapists see it, is that many other parallel regulatory hurdles have been erected in the meantime. The US Food and Drug Administration (FDA) must approve clinical trials of gene therapy in humans. And institutions have their own biosafety committees and institutional review boards.

Naturally, the RAC disagrees from time to time with the findings of the other scrutineers. When it does, the delays can drag on.

Enough, said the American Society of Gene and Cell Therapy last March. It told the NIH that in recent decades, more than 1,000 genetherapy clinical trials have been conducted. The worst fears of the public - that gene therapy would lead to alterations of the human genome, or to the release of genetically modified super microbes have not come to pass. The society told the NIH that the RAC should no longer review individual gene-therapy protocols, and should instead "identify new areas of research that require a public forum for discussion and review".

The gene-therapy field is not free from the risk of adverse events, but the RAC has never claimed to be able to prevent them. Instead, it has had a crucial role in helping the field to learn from setbacks.

After the death of US teenager Jesse Gelsinger in a gene-therapy trial in 1999, for instance, the RAC adopted rules that compel investigators to report all serious adverse events that occur during gene-therapy trials. When children who had been cured of severe combined immunodeficiency by gene therapy developed leukaemia in 2001, the RAC

investigated how gene therapy might have contributed to the problem and recommended action to stop it recurring.

Researchers feel strongly that RAC review of individual protocols no longer serves an important purpose, and they resent being at the mercy of the committee's ability to call them out on questions that they often perceive as tangential to their research. Yet it is a tricky time to argue that any public-review process in medical research should be scaled

"It is a tricky time to argue that any publicreview process in medical research should bescaled back." back. Witness the storm of public outrage in the past decade over pharmaceutical companies' failure to report side effects of drugs for conditions from diabetes to depression (see Nature 431, 122-124; 2004). Long after these drugs were approved, it was revealed that their sponsors had held back crucial information about their safety.

Gene-therapy clinical trials have proceeded with an unusually high degree of openness, and that has been crucial in helping the field to gain public confidence and acceptance. Such a role should be emulated in other areas of research, rather than eliminated. Apart from the RAC review, none of the oversight required for gene therapy is public. FDA review includes public meetings, but the formal review process allows investigators to keep many of their data secret.

The question is how to preserve the openness that the RAC has enabled without bogging down progress. Perhaps now is the right time to scale back the RAC's purview, but it will be imperative to do so while maintaining the committee's moral authority. That position may feel burdensome, but without it, the field could not have got to where it is today.
ANNOUNCEMENT

\section{Nature papers enhanced}

A $s$ the requirements for data presentation in research papers have grown, Nature's space limitations have remained tight, so more and more essential displayed information has been relegated inappropriately to our Supplementary Information sections. Hard on the heels of our relaxation of constraints on our online Methods sections (see Nature 496, 398; 2013), we are now significantly increasing the number of figures integral to the paper in its online and PDF versions. From July, Nature will introduce a new component to its research papers. This new category, called Extended Data (see go.nature.com/tp4vu3), will provide the online reader with immediate access to many display items (figures and tables) previously buried in the Supplementary Information PDF. From now on, most papers submitted to Nature can take advantage of this enhancement.

Extended Data display items will be referred to in the print version of the paper, but will be available only online (as is also the case with our full Methods sections). Individual Extended Data display items will be easily accessible by clicking on a call-out in the HTML version of the paper, generating a pop-up box containing the display item and its accompanying legend. Furthermore, the Extended Data display items will be appended to the end of the online PDF, so that the print paper, full Methods section and Extended Data section will be available in one document (see go.nature.com/gb5p6r for a breakdown of the composition of a Nature research paper).

Extended Data will not normally contain more than ten individual display items (figures and tables) in addition to the limits set for the printed version of the paper (typically four and five display items for Letters and Articles, respectively). Authors are encouraged to combine appropriate Extended Data figures into multi-panelled figures in order to meet this limit. Each display item should fit onto one page, ideally with its legend or footnote directly below.

The Extended Data display items will be peer reviewed but, like current Supplementary Information, will not be edited in-house. At final submission the Extended Data display items should be generated at the same quality as the figures for the print paper, although there will be differences in formatting (see go.nature.com/zmitgz for a full formatting guide).

Extended Data display items can be used to present essential information relating to the Methods section.

The Supplementary Information section will remain as part of the online-only content, comprising material directly relevant to the conclusion of a paper that cannot be included in the printed version for reasons of space or medium (for example, video clips or sound files). However, this section should no longer contain figures or tables unless there is an exceptional justification (for example, if information is best presented in an Excel file).

From the beginning of July, editors will ask authors who have been invited to revise their papers after the first round of peer review to reformat their papers for consistency with Extended Data. In addition, editors will identify papers at later stages in the editorial process (up to and including the final revision) that might be easily reformatted to include Extended Data display items, and invite authors to revise their papers accordingly. Eventually, all new submissions to Nature will be required to comply with this formatting of research papers. The result will be a higher standard of data presentation within the online-only versions of the paper, which will be to the benefit of our readers. 